\title{
Effects of Blue and Red Lights on Gibberellin Metabolism in Tomato Seedlings
}

\author{
Satoshi Matsuo ${ }^{1 *}$, Kenji Nanya ${ }^{2 * *}$, Shunsuke Imanishi ${ }^{1}$, Ichiro Honda ${ }^{1 * * *}$ and Eiji Goto ${ }^{2}$ \\ ${ }^{1}$ Institute of Vegetable and Floriculture Science, NARO, Tsu 514-2392, Japan \\ ${ }^{2}$ Graduate School of Horticulture, Chiba University, Matsudo 271-8510, Japan
}

Light quality is an important environmental factor that regulates stem length of the tomato (Solanum lycopersicum). Previously, we showed that the stem length of tomato seedlings grown under red (R) light emitting diodes (LEDs) was significantly longer than that of seedlings grown under blue (B) LEDs or a mixture of B and R LEDs; further, the light intensity of B LED negatively correlated with the stem length. This study aimed to elucidate the mechanism of how $B$ and $R$ lights affect stem elongation. We analyzed the levels of gibberellins (GAs) and the expression of genes associated with their metabolism in tomato seedlings grown under different $B$ and $R$ light conditions. The level of bioactive $\mathbf{G A}, \mathbf{G A}_{4}$, was significantly higher in the seedlings grown under R LED than in those grown under other light conditions. In addition, an increase in the $B$ to $R$ light ratio increased the transcript level of the GA inactivation enzyme gene, SlGA2ox 7 . Moreover, the transcript level of $S I G A 20 x 7$ increased with the intensity of B light, and was negatively correlated with the stem length of the seedlings. These results indicated that the B light intensity controlled GA inactivation of the seedlings, and endogenous GA contents may affect stem elongation. Further, we found that the transcript level of the GA biosynthesis enzyme gene, SlGA3ox3, in the seedlings grown under R LED was significantly higher than that under other light conditions. This could be due to depletion of $B$ light and suggests that GA biosynthesis may be involved in the stem elongation of seedlings grown under low B light conditions.

Key Words: gibberellin 2-oxidase, gibberellin 3-oxidase, light emitting diode, light quality, stem elongation.

\section{Introduction}

The tomato (Solanum lycopersicum) is an important vegetable crop of commercial value worldwide. In tomato cultivation, transplanting high-quality seedlings is essential to stabilize fruit yield. Recently, a closed plant production system (CPPS) for seedling cultivation with artificial light has been used as a commercial nursery system; this system enables the efficient production of high-quality seedlings (Kozai, 2013; Ohyama et al., 2003). For effective seedling production in a limited space, seedlings are grown at a high planting density in

Received; April 10, 2018. Accepted; August 29, 2018.

First Published Online in J-STAGE on October 20, 2018.

This work was supported by a grant "Elucidation of biological mechanisms of photo response and development of advanced technologies utilizing light" funded by the Ministry of Agriculture, Forestry and Fisheries of Japan. Part of this study was presented at the 2013 spring meeting of the Japanese Society for Horticultural Science.

* Corresponding author (E-mail: mtosts@affrc.go.jp).

** Present address: Northern Advancement Center for Science and Technology, Sapporo 062-0052, Japan.

*** Present address: Department of Biotechnology, Maebashi Institute of Technology, Maebashi 371-0816, Japan. a CPPS. Under high planting density conditions, the stems of seedlings often elongate too much, resulting in spindly growth. The use of these seedlings cause problems such as broken stems during transplanting, so it is necessary to prevent spindly growth in seedling production. The desirable morphologies of seedlings depend on the objective. For example, the control of hypocotyl and stem length is important for grafted seedling production because the use of scission and rootstock plants with uniform size is necessary to improve work efficiency in grafting operations (Singh et al., 2017). For these reasons, controlling plant morphology is important in seedling production, and it is necessary to develop an effective method to manipulate plant morphology in a CPPS.

Light quality is one of the important environmental factors that controls plant growth and development. The relative quantum yield of photosynthesis in the tomato is highest at red (R) wavelengths $(600-700 \mathrm{~nm}$; McCree, 1972). Thus, the use of $\mathrm{R}$ light is the most effective way to drive photosynthesis. Additionally, previous studies indicated that some blue (B) light is essential for normal growth and development of many plant species (Britz and Sager, 1990; Brown et al., 
1995; Yorio et al., 2001). B light is recognized by photoreceptors such as cryptochromes and phototropins, and these photoreceptors play important roles in photomorphogenesis (Lin, 2002). Several studies have revealed the effects of $\mathrm{B}$ and $\mathrm{R}$ lights on the growth of the tomato (Hernández et al., 2016; Nanya et al., 2012; Wollaeger and Runkle, 2014, 2015) and showed that seedlings grown under B light have shorter stems than those grown under $\mathrm{R}$ light. These results indicate that manipulation of $\mathrm{B}$ and $\mathrm{R}$ lights can control the stem length of tomato seedlings. Recently, the use of light emitting diodes (LEDs) has increased in CPPS, and B and R LEDs are used as the light sources for plant cultivation. Understanding the mechanisms underlying the effects of $\mathrm{B}$ and $\mathrm{R}$ lights on the stem length of the tomato may allow effective control of light conditions in a CPPS and improve the productivity of high-quality tomato seedlings.

Gibberellins (GAs) are plant hormones that play important roles in photomorphogenesis processes such as de-etiolation, seed germination, and shade avoidance responses (García-Martinez and Gil, 2002). During deetiolation, stem elongation was suppressed by light irradiation in many plants. Several studies showed that this suppression was mediated by the reduction of GA action and both $\mathrm{B}$ and $\mathrm{R}$ photoreceptors were involved in the transcriptional regulation of GA metabolic enzyme genes (Foo et al., 2006; Hirose et al., 2012; Zhao et al., 2007). Thus, manipulation of $B$ and $R$ lights can regulate GA metabolism and influence plant morphology. However, little is known about the effects of the ratio or intensity of $\mathrm{B}$ and $\mathrm{R}$ lights on the regulation of GA metabolism in tomato seedlings.

Among GAs, $\mathrm{GA}_{1}$ and $\mathrm{GA}_{4}$ have been recognized as the major endogenous active forms in plants (Fig. 1). They are synthesized from trans-geranylgeranyl diphosphate (GGDP), which is converted to ent-kaurene in plastids (Hedden and Thomas, 2012; Yamaguchi, 2008). Subsequently, ent-kaurene is converted to $\mathrm{GA}_{12}$ by cytochrome $\mathrm{P} 450$ monooxygenases located in the endoplasmic reticulum. Further steps are divided into two routes: the non-13-hydroxylation and early-13hydroxylation pathways. In both pathways, gibberellin 20-oxidase (GA20ox) and gibberellin 3-oxidase (GA3ox), which belong to 2-oxoglutarate-dependent dioxygenases, catalyze the series of oxidative reactions in the cytosol and synthesize the active forms of GAs. Inactivation of GAs mainly occurs via $2 \beta$-hydroxylation, and gibberellin 2-oxidase (GA2ox), a 2-oxoglutaratedependent dioxygenase, catalyzes this reaction. Endogenous levels of active GAs are considered to be regulated by the expression of GA20ox, GA3ox, and GA2ox genes (Yamaguchi, 2008).

In a previous study, we investigated the effects of exposure to $\mathrm{B}$ and $\mathrm{R}$ lights from LEDs on the growth of tomato seedlings and found that the $\mathrm{B}$ light intensity controlled the stem length (Nanya et al., 2012). In this

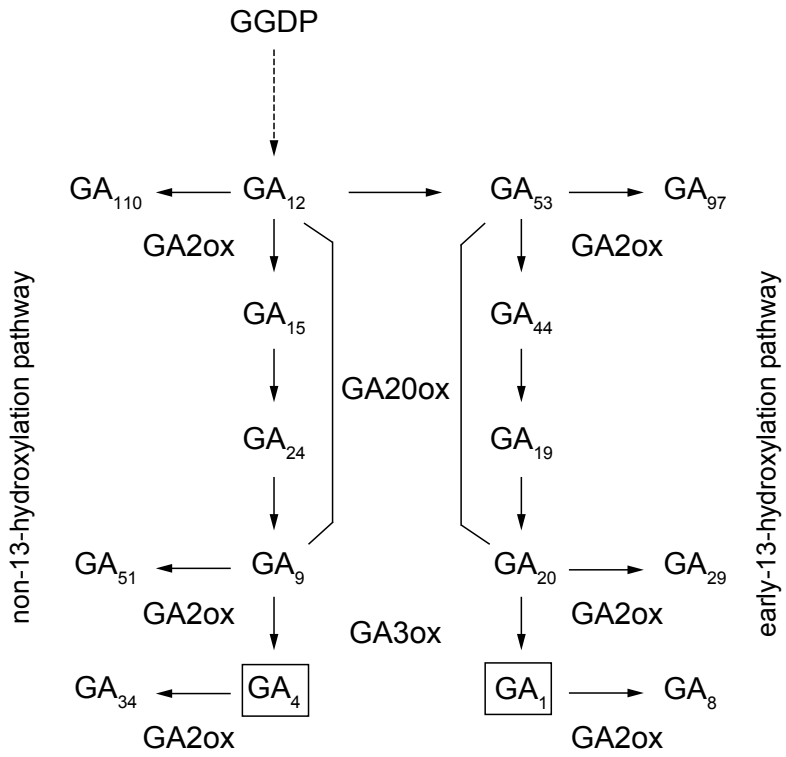

Fig. 1. The major gibberellin (GA) metabolic pathway in plants. GA2ox: GA 2-oxidase, GA20ox: GA 20-oxidase, GA3ox: GA 3-oxidase, GGDP: trans-geranylgeranyl diphosphate. $\mathrm{GA}_{1}$ and $\mathrm{GA}_{4}$ are active forms of GAs.

study, we determined the effects of $\mathrm{B}$ and $\mathrm{R}$ lights on the regulation of GA metabolism in tomato seedlings. First, we quantified the endogenous concentrations of GAs and indole-3-acetic acid (IAA) in tomato seedlings under different $\mathrm{B}$ to $\mathrm{R}(\mathrm{B} / \mathrm{R})$ ratio light conditions. Next, we analyzed the transcript levels of genes involved in GA metabolism. Finally, we determined the effects of different light intensities of $\mathrm{B}$ and $\mathrm{R}$ lights on the regulation of GA metabolic enzyme genes.

\section{Materials and Methods}

\section{Plant materials and growth conditions}

The detailed growth conditions used were those described in Nanya et al. (2012). Briefly, tomato seeds (S. lycopersicum) 'Reiyo' (Sakata Seed Co., Kanagawa, Japan) were sown in culture soil (Yanmar Agricultural Machinery Manufacturing Co., Ltd., Osaka, Japan) and kept under dark conditions for three days, and then grown under white fluorescent lamps with $16 / 8 \mathrm{~h}$ light/ dark period at $26 / 19^{\circ} \mathrm{C}$ (light/dark) for seven days in a controlled environment room (Nishimura et al., 2001). Plants were transferred under LED panels (Ushio Lighting Inc., Tokyo, Japan) under the same conditions as mentioned above. The effects of the $\mathrm{B} / \mathrm{R}$ ratio on the growth of tomato seedlings were determined by irradiating plants at a photosynthetic photon flux density (PPFD) of $150 \mu \mathrm{mol} \cdot \mathrm{m}^{-2} \cdot \mathrm{s}^{-1}$ of B (Fig. S1; peak wavelength, $450 \mathrm{~nm}$ ) LED (B150), R (Fig. S1; peak wavelength, $660 \mathrm{~nm}$ ) LED (R150), and mixture of $\mathrm{B}$ and $\mathrm{R}$ LEDs with two different $\mathrm{B} / \mathrm{R}$ ratios $(0.4$; B45R $105,1.0$; $B 75 R 75)$. The effects of $B$ and $R$ light intensities on the growth of tomato seedlings were investigated by fixing the PPFD of B or R lights at $75 \mu \mathrm{mol} \cdot \mathrm{m}^{-2} \cdot \mathrm{s}^{-1}$ and that 
of the other light at 25,50 , and $75 \mu \mathrm{mol} \cdot \mathrm{m}^{-2} \cdot \mathrm{s}^{-1}$ (B25R75, B50R75, B75R75, and B75R25, B75R50, B75R75). 15-day-old seedlings were harvested $4 \mathrm{~h}$ after dawn and used to quantify plant hormones and analysis of gene expression. In the $\mathrm{B} / \mathrm{R}$ ratio experiment, we used the different samples described in Nanya et al. (2012) for GA quantification and gene expression analysis. The stem lengths of the samples are shown in Fig. S2. In the B and R light intensity experiments, we used the same samples as those described in Nanya et al. (2012) for gene expression analysis and the detailed data for those seedlings was described there.

\section{Quantification of plant hormones}

About $2 \mathrm{~g}$ of whole aerial tissues of tomato seedlings were homogenized in liquid nitrogen and placed in $10 \mathrm{ml}$ of cold $\left(-20^{\circ} \mathrm{C}\right)$ methanol/water/formic acid $(15: 4: 1, \mathrm{v} / \mathrm{v} / \mathrm{v})$. The $\left[{ }^{2} \mathrm{H}_{2}\right] \mathrm{GA}_{1},\left[{ }^{2} \mathrm{H}_{2}\right] \mathrm{GA}_{4},\left[{ }^{2} \mathrm{H}_{2}\right] \mathrm{GA}_{8}$, $\left[{ }^{2} \mathrm{H}_{2}\right] \mathrm{GA}_{9},\left[{ }^{2} \mathrm{H}_{2}\right] \mathrm{GA}_{19},\left[{ }^{2} \mathrm{H}_{2}\right] \mathrm{GA}_{20},\left[{ }^{2} \mathrm{H}_{2}\right] \mathrm{GA}_{24},\left[{ }^{2} \mathrm{H}_{2}\right] \mathrm{GA}_{34}$ (OlchemIm, Olomouc, Czech Republic), and $\left[{ }^{13} \mathrm{C}_{6}\right] \mathrm{IAA}$ (Cambridge Isotope Laboratories Inc., Tewksbury, MA, USA) were used as internal standards. Extraction was performed overnight at $-20^{\circ} \mathrm{C}$. The samples were centrifuged, and supernatants were collected. Solids were re-extracted for $30 \mathrm{~min}$ in $10 \mathrm{ml}$ of the same extraction solution, and supernatants were collected. The combined extract was applied to an Oasis HLB column (200 mg; Waters, Milford, MA, USA) that had been equilibrated with $1 \mathrm{M}$ formic acid. The eluate was evaporated and then dissolved in $5 \mathrm{ml}$ of $1 \mathrm{M}$ formic acid and applied to an Oasis MCX column (150 mg; Waters) that had also been equilibrated with $1 \mathrm{M}$ formic acid. After the column was washed with $5 \mathrm{ml}$ of $1 \mathrm{M}$ formic acid, GAs and IAA were eluted with $10 \mathrm{ml}$ of $100 \%$ methanol. The eluate was evaporated, dissolved in $3 \mathrm{ml}$ of $1 \%$ acetic acid, and applied to an Oasis WAX column (60 mg; Waters) that had been equilibrated with $1 \%$ acetic acid. After the column was washed with $3 \mathrm{ml}$ of $1 \%$ acetic acid and $3 \mathrm{ml}$ of $100 \%$ methanol, hormones were eluted with $6 \mathrm{ml}$ of $80 \%$ methanol containing $1 \%$ acetic acid. The eluate was evaporated, dissolved in methanol/chloroform/acetic acid (50/49/1, $\mathrm{v} / \mathrm{v} / \mathrm{v})$, and applied to a Sep-Pak silica column $(500 \mathrm{mg}$, Waters) that had been equilibrated with chloroform containing $1 \%$ acetic acid. The flow through was collected, and the column was further washed with $6 \mathrm{ml}$ of metha$\mathrm{nol} / \mathrm{chloroform} /$ acetic acid $(50 / 49 / 1, \mathrm{v} / \mathrm{v} / \mathrm{v})$. The combined eluate was evaporated and dissolved in water/ methanol/acetic acid $(89 / 10 / 0.1, \mathrm{v} / \mathrm{v} / \mathrm{v})$. The purified samples were analyzed using high-performance liquid chromatography (HPLC)/tandem quadrupole mass spectrometry (MS/MS), which consisted of a Prominence 20A Series HPLC (Shimadzu, Kyoto, Japan) and a 3200 QTrap LC/MS/MS System (AB Sciex, Framingham, MA, USA), by using an electrospray interface. The GAs and IAA were quantified according to Kotoda et al. (2016) and Honda et al. (2017). Three bio- logical replicates were analyzed for all samples.

\section{Quantitative real-time PCR analysis}

The sequences of tomato GA metabolic enzyme genes were obtained from the NCBI (http:// www.ncbi.nlm.nih.gov) and SOL Genomics Network (https://solgenomics.net) databases. The transcripts were quantified using quantitative real-time PCR, as described by Matsuo et al. (2012). Total RNA was extracted from whole aerial tissues of tomato seedlings by using an RNeasy Plant Mini Kit (Qiagen, Venlo, Netherlands). DNase treatment was performed using an RNase Free DNase I kit (Qiagen). First-strand cDNA synthesis was performed using a Transcriptor First Strand cDNA Synthesis Kit (Roche Diagnostics, Basel, Switzerland). Quantitative real-time PCR was performed using a LightCycler 480 SYBR Green I Master (Roche Diagnostics). The SAND gene (Solyc03g115810) was used as reference (ExpósitoRodríguez et al., 2008). The primer pair information is shown in Table S1. The PCR reactions were performed on a LightCycler 480 system (Roche Diagnostics). Three to four biological replicates were analyzed for all samples.

\section{Results}

Effects of blue to red light ratio on the GA content of tomato seedlings

In our previous study, the stem length of tomato seedlings grown under R LED (R150) was found to be significantly higher than that of seedlings grown under B (B150) or a mixture of B and R LEDs (B45R105 and B75R75; Nanya et al., 2012). To determine the mechanisms underlying the effects of $\mathrm{B}$ and $\mathrm{R}$ lights on the control of tomato stem length, we measured the endogenous levels of two growth-promoting plant hormones, GAs and IAA, in tomato seedlings grown under different $\mathrm{B} / \mathrm{R}$ ratio conditions (Fig. 2). As reported by Asahina et al. (2007), GAs of both early-13hydroxylation and non-13-hydroxylation pathways were detected in tomato seedlings (Fig. 2A). The levels of $\mathrm{GA}_{4}$ (the bioactive $\mathrm{GA}$ of the non-13-hydroxylation pathway) were significantly higher in R150 $\left(0.42 \mathrm{ng} \cdot \mathrm{g}^{-1}\right.$ fresh weight (FW)) than in the other light conditions. The $\mathrm{GA}_{4}$ concentrations in B45R105 $\left(0.27 \mathrm{ng} \cdot \mathrm{g}^{-1} \mathrm{FW}\right)$, B75R75 (0.24 $\left.\mathrm{ng} \cdot \mathrm{g}^{-1} \mathrm{FW}\right)$, and B150 (0.26 ng. $\left.\mathrm{g}^{-1} \mathrm{FW}\right)$ did not differ significantly. The levels of $\mathrm{GA}_{34}$ (the metabolite of $\mathrm{GA}_{4}$ ) were also significantly higher in R150 (3.27 ng $\cdot \mathrm{g}^{-1} \mathrm{FW}$ ) than in other light conditions and the lowest in B75R75 (1.13 $\left.\mathrm{ng} \cdot \mathrm{g}^{-1} \mathrm{FW}\right)$. The concentrations of $\mathrm{GA}_{24}$ (the precursor of $\mathrm{GA}_{4}$ ) and $\mathrm{GA}_{1}$ (the bioactive GA of early-13-hydroxylation pathway) did not differ among the light conditions. The level of $\mathrm{GA}_{8}$ (the metabolite of $\mathrm{GA}_{1}$ ) was significantly lower in B75R75 $\left(1.20 \mathrm{ng} \cdot \mathrm{g}^{-1} \mathrm{FW}\right)$ than in R150 (1.96 $\left.\mathrm{ng} \cdot \mathrm{g}^{-1} \mathrm{FW}\right)$ and $\mathrm{B} 150\left(1.88 \mathrm{ng} \cdot \mathrm{g}^{-1} \mathrm{FW}\right)$. The concentration of $\mathrm{GA}_{19}$ (the precursor of $\mathrm{GA}_{1}$ ) was significantly lower in R150 than 
A

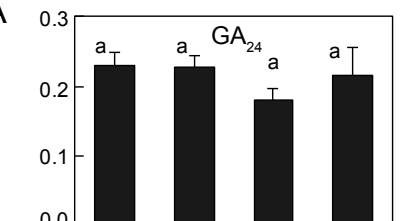

$\sum_{0.5}$
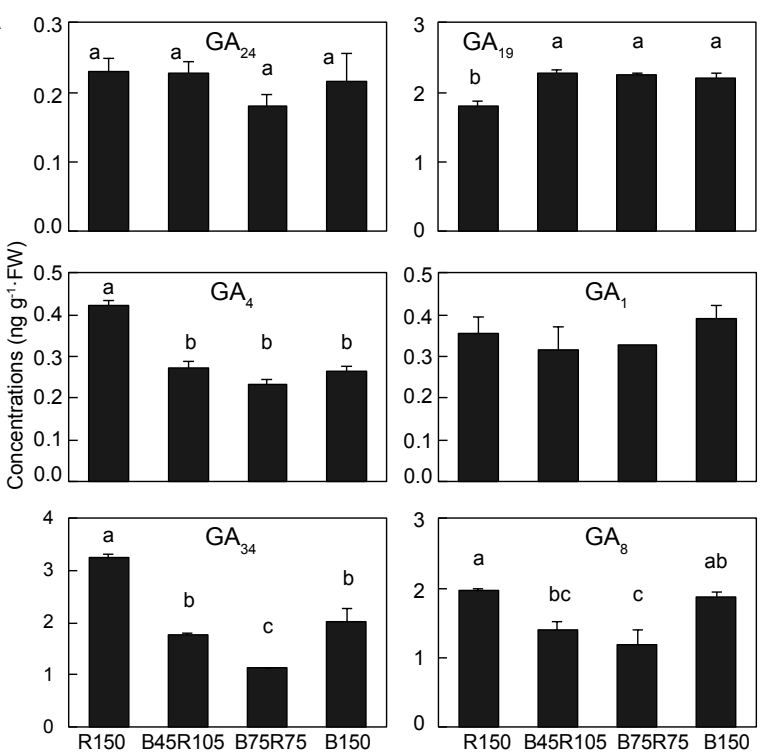

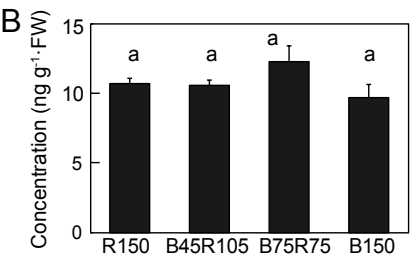

Fig. 2. Endogenous levels of (A) gibberellins (GAs) and (B) indole-3-acetic acid (IAA) in tomato seedlings grown under different blue to red ratio conditions (mean $\pm \mathrm{SE}$ ). The results show the average of three samples except $\mathrm{GA}_{1}$ in $\mathrm{B} 75 \mathrm{R} 75$, which is the average of two samples, because one sample could not be quantified due to impurity co-migration. Different letters indicate significant differences determined by the TukeyKramer test for multiple comparisons at $P<0.05$.

in other light conditions. $\mathrm{GA}_{9}$ and $\mathrm{GA}_{20}$ could not be quantified because of low levels or co-migration of impurities (data not shown). Among the GAs, the concentrations of $\mathrm{GA}_{4}$ and $\mathrm{GA}_{34}$ were positively correlated with the stem length of tomato seedlings (Fig. S3A, B). No significant differences were noted in the IAA contents among the light conditions (Fig. 2B). These results suggested that the contents of GAs, especially that of $\mathrm{GA}_{4}$, may be responsible for the differences in the stem length of tomato seedlings grown under different light conditions.

\section{Effects of blue to red light ratio on the expression of GA} metabolic enzyme genes

To analyze the relationship between GA metabolism and light quality, we investigated the expression profiles of GA metabolic enzyme genes in seedlings grown under different B/R ratio conditions (Fig. 3, Table S2). Four GA20ox (SlGA20oxl to 4), two GA3ox (SlGA3oxl and 2), and eleven GA2ox (SlGA2ox1 to 11) genes have been reported in the tomato (Chen et al., 2016; Reberts et al., 1999; Serrani et al., 2008). We found three additional GA20ox-like (Solyc06g050110, Solyc09g009110,
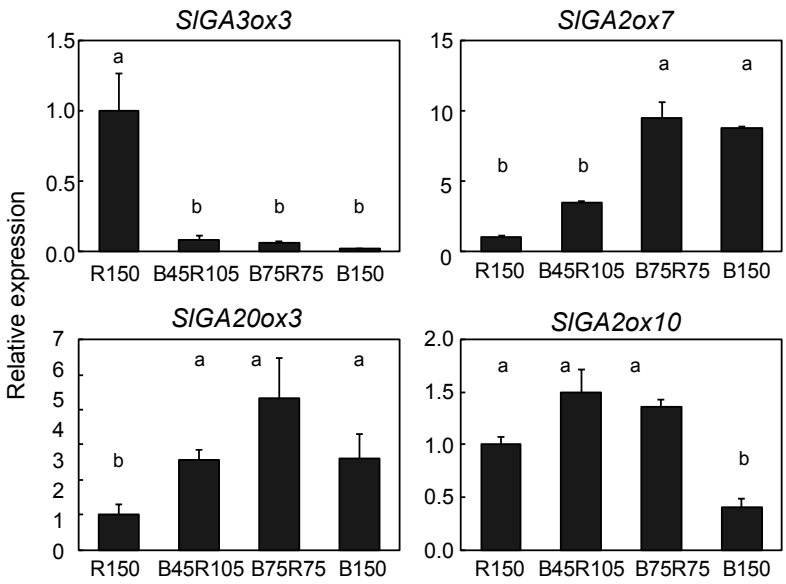

Fig. 3. Expression of gibberellin (GA) metabolic enzyme genes in tomato seedlings grown under different blue to red ratio conditions (mean $\pm \mathrm{SE}, \mathrm{n}=3$ ). Real-time PCR was performed using cDNA prepared from seedlings grown under different blue to red light ratio conditions. Different letters indicate significant differences determined by Tukey-Kramer test for multiple comparisons at $P<0.05$.

and Solyc12g013780) and three GA3ox-like (Solyc01g058250, Solyc00g007180, and Solyc05g052740) sequences in the tomato genome. Among the GA metabolic enzyme genes, the transcript levels of SlGA3ox-like (Solyc01g058250), SlGA2ox7 (Solyc02g080120), SlGA2ox10 (Solyc06g082030), and SlGA20ox3 (Solyc1lg072310) were affected by the changes in the B/R ratio (Fig. 3). Because SlGA3ox genes except for SlGA3oxl and SlGA3ox2 were not named previously, we designated them Solyc01g058250 as $S l G A 3 o x 3$ in this study. The transcript level of SlGA3ox3 was clearly higher in R150 than in the other light conditions. The expression of SlGA20x7 was significantly lower in R150 and B45R105 than in B75R75 and B150. The expression of SlGA20ox3 was significantly lower in R150 than in the other light conditions. The expression of SlGA2ox10 was significantly lower in B150 than in the other light conditions.

Effects of blue and red light intensities on the expression of GA metabolic enzyme genes

To further analyze the relationships between light quality and GA metabolism, we investigated the effects of $\mathrm{B}$ and $\mathrm{R}$ light intensities on the expression of GA metabolic enzyme genes of tomato seedlings. In our previous study, the stem length of tomato seedlings was found to decrease with increasing B light intensity, whereas no significant differences in stem length were observed with increasing R light intensity (Nanya et al., 2012). The expression of $S l G A 2 o x 7$ increased with the B light intensity, whereas the transcript level of SlGA2ox7 did not differ significantly among the different $\mathrm{R}$ light intensity conditions (Fig. 4A). The transcript level of SIGA20x7 was negatively correlated with the stem length of tomato seedlings $\left(R^{2}=0.85, P=0.009\right.$, 
A

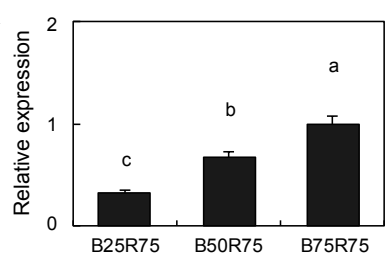

B

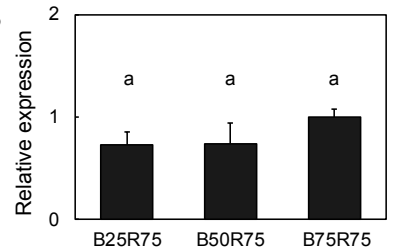

C

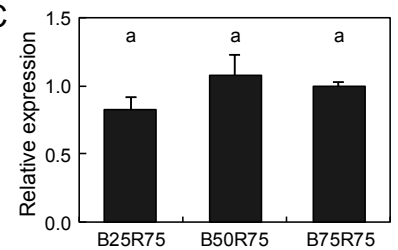

$\mathrm{D}$

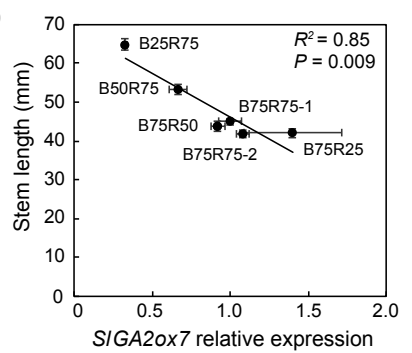

Fig. 4. Expression of gibberellin (GA) metabolic enzyme genes in tomato seedlings grown under different conditions of blue and red light intensity. Expression of (A) $S l G A 2 o x 7$, (B) $S l G A 3 o x 3$, and (C) SlGA2ox10 (mean $\pm \mathrm{SE}, \mathrm{n}=3-4)$. Real-time PCR was performed using cDNA prepared from seedlings grown under different conditions of blue and red light intensity. Different letters indicate significant differences determined by the TukeyKramer test for multiple comparisons at $P<0.05$. (D) Relationship between the relative expression level of $S l G A 20 x 7$ and stem length (Nanya et al., 2012) of tomato seedlings grown under different blue (B25R75, B50R75, and B75R75-1) and red (B75R25, B75R50, and B75R75-2) light intensities. Means $( \pm \mathrm{SE})$ of SlGA2ox7 expression level $(\mathrm{n}=3)$ and stem length $(n=6)$ were used to generate the scores plot.

Fig. 4D). The expression of SlGA3ox3 in B75R75 was significantly higher than that in B75R25 and B75R50, but did not differ significantly among the different $\mathrm{B}$ light intensity conditions (Fig. 4B). The expression of SlGA2ox10 in B75R75 was significantly higher than that in B75R25 and B75R50, but did not differ significantly among the different B light intensity conditions (Fig. 4C). There was no correlation between the transcript levels of these two genes and the stem length (SlGA3ox3 $R^{2}=0.14, P=0.47$; SlGA2ox10 $R^{2}=0.01$, $P=0.89$ ). No significant differences were noted in the expression level of SlGA20ox3 among the different B and $\mathrm{R}$ light intensity conditions (data not shown).

\section{Discussion}

To elucidate the mechanism of light quality on tomato stem elongation, we determined the contents of GAs and transcripts of GA metabolic enzyme genes in tomato seedlings grown under different $\mathrm{B}$ and $\mathrm{R}$ light conditions. The concentrations of GAs showed different patterns among the different $\mathrm{B} / \mathrm{R}$ ratio conditions (Fig. 2A). The levels of bioactive $\mathrm{GA}_{4}$ in $\mathrm{R} 150$ were significantly higher than those in other light conditions, whereas the $\mathrm{GA}_{1}$ content was not affected by the changes in the $B / R$ ratio. Several studies showed the dominance of GAs of non-13-hydroxylation pathways in young tomato plants (Asahina et al., 2007; Nakaune et al., 2012). Thus, GAs of the non-13-hydroxylation pathway may have dominant roles in response to the changes in the $\mathrm{B} / \mathrm{R}$ ratio compared to GAs of the early-13-hydroxylation pathway. The high correlation between endogenous $\mathrm{GA}_{4}$ content and stem length of seedlings suggests that $\mathrm{GA}_{4}$ plays an important role in stem elongation (Fig. S3A). The higher transcript level of SlGA3ox3 in R150 may be related to the higher contents of $\mathrm{GA}_{4}$ (Figs. 2A and 3). In addition, we observed that the transcript levels of SlGA2ox7 in R150 and B45R105 and that of SlGA2ox10 in B150 were lower than those in the other light conditions. Tomato GA2ox family proteins are classified into three subgroups: I, II, and III. Subgroups I and II catalyze $\mathrm{C}_{19}$ type GAs such as $\mathrm{GA}_{1}, \mathrm{GA}_{4}, \mathrm{GA}_{9}$ and $\mathrm{GA}_{20}$, whereas subgroup III catalyzes $\mathrm{C}_{20}$ type GAs such as $\mathrm{GA}_{12}$ and $\mathrm{GA}_{53}$ (Fig. 1; Chen et al., 2016). As per the amino acid sequences, both SIGA2ox7 and SIGA2ox10 belong to subgroup III. Thus, the expression of SlGA2ox7 and SlGA2ox10 may not directly influence the levels of $\mathrm{GA}_{34}$, and the higher level of $\mathrm{GA}_{34}$ in $\mathrm{R} 150$ may be a result of the higher level of $\mathrm{GA}_{4}$. SlGA2ox7 expression increased with increasing B light intensity, but not of $\mathrm{R}$ light (Fig. 4A). These results indicated that this gene expression was controlled by the B light intensity. The transcript levels of SlGA2ox10 and SlGA3ox3 increased with increasing $\mathrm{R}$ light intensity (Fig. 4B, C). Thus, the expression of these genes might be controlled by $\mathrm{R}$ light intensity. However, markedly high levels of $\mathrm{GA}_{4}$ and the SlGA3ox3 transcript were observed only in R150 (Figs. 2A and 3). The expression of SlGA20ox3 in R150 was lower than those in other light conditions (Fig. 3). Some GA biosynthesis enzyme genes are known to be regulated by endogenous GA levels through feedback regulation (Hedden and Thomas, 2012). Thus, the lower expression of SlGA20ox3 may be caused by the higher endogenous levels of GAs in R150. These results suggest that SlGA2ox7, SlGA2ox10, and SlGA3ox3 are involved in the regulation of GA metabolism under B and $\mathrm{R}$ light conditions. Among the three genes, the expression of SlGA2ox7 was negatively correlated with the stem length of seedlings (Fig. 4D), whereas there was no correlation between the expression of the two other genes and stem length. Therefore, B light-induced SlGA2ox7 expression may be the most important factor in controlling the stem length of seedlings. In plants, 
the expression of GA metabolic enzyme genes changes during their life cycle and each gene family member has distinct and redundant roles in different developmental processes (Hedden and Thomas, 2012; Mitchum et al., 2006; Rieu et al., 2008). Thus, it is possible that other genes have important roles in stem elongation in other developmental stages of tomato seedlings. Future studies, such as time-course analysis of GAs and GA metabolic enzyme genes are needed to better understand the effects of light quality on GA metabolism in tomato seedling development.

The effects of B light on GA metabolism has been reported in de-etiolating seedlings of several plants (Foo et al., 2006; Hirose et al., 2012; Zhao et al., 2007). During de-etiolation, GA contents were rapidly decreased by $\mathrm{B}$ light irradiation via the upregulation of several GA2ox genes. Thus, the mechanism of B light irradiation on light-grown tomato seedlings resembles that of de-etiolating seedlings of other plants. Several recent reports suggest that the $\mathrm{B}$ light intensity influences the shade avoidance responses of Arabidopsis (de Wit et al., 2016; Pedmale et al., 2016). When plants were placed under the shade of neighboring plants, various developmental responses, including stem elongation, were induced. Plants sense the low R/FR ratio light via phytochromes and control the endogenous levels and responsiveness of plant hormones (Casal, 2012; Kurepin and Pharis, 2014). In addition to R light, B light is absorbed by photosynthetic pigments, and thus the $\mathrm{B}$ light intensity decreases under vegetative tissues of neighboring plants. Plants sense low B light (LBL) as well as low R/FR ratio light, and LBL induces various responses, including stem elongation (de Wit et al., 2016; Pedmale et al., 2016), which are mediated by B light photoreceptors. Several studies showed that the suppression of the B light photoreceptor gene, cryptochrome 1, increased the stem length in the tomato (Ninu et al., 1999; Weller et al., 2001). In addition, the overexpression of another B light photoreceptor gene, cryptochrome 2, repressed stem elongation in the tomato (Giliberto et al., 2005). These results suggest that irradiation with B light is indispensable to suppress spindly growth of the tomato. Stem length of tomato seedlings grown under R150 was significantly higher than that of seedlings grown under $\mathrm{B} 150$ or two $\mathrm{B} / \mathrm{R}$ ratio conditions (Nanya et al., 2012). The marked stem elongation of tomato seedlings grown under R LED has also been reported previously (Wollaeger and Runkle, 2014, 2015). Because light intensities were the same among the growth conditions in our $\mathrm{B} / \mathrm{R}$ ratio experiment, the longer stems in R150 are thought to be a result of B light depletion, and a high endogenous GA content may be involved in stem elongation. The expression of SlGA3ox3 in R150 was more than 10 times higher than that in other light conditions, and irradiation of B light decreased its expression (Fig. 3). Because there was no correlation between the SlGA3ox 3 transcript level and stem length, this gene may play a role in stem elongation under the LBL condition in particular.

Stem length of tomato seedlings grown under B150 was significantly higher than that of seedlings grown under B75R75 (Fig. S2; Nanya et al., 2012). The SlGA2ox10 transcript level in B150 was lower than that in B75R75 (Fig. 3), and this may be the cause of the longer stems of B150 than those of B75R75. These results indicated that only the B light intensity was not involved in the suppression of stem elongation. During de-etiolation, $\mathrm{R}$ light irradiation induced the expression of GA2ox genes and suppressed stem elongation (Foo et al., 2006; Hirose et al., 2012; Zhao et al., 2007). In addition, several studies showed that the stem length of tomato phytochrome mutants was longer than that of the wild-type plants (van Tuinen et al., 1995; Weller et al., 2000). Thus, the depletion of $\mathrm{R}$ light also promotes stem elongation, and SlGA2ox10 may be involved in the low $\mathrm{R}$ light response. The effects of low $\mathrm{R}$ light on the increase in active GAs and stem length were low under our experimental conditions. This may be due to the B light-induced reduction of active GAs. Thus, the effect of $\mathrm{R}$ light on the regulation of endogenous GA contents may be less than that of $\mathrm{B}$ light.

In conclusion, we investigated the effects of $\mathrm{B}$ and $\mathrm{R}$ lights on the regulation of GA metabolism in tomato seedlings and found that the B light-induced SlGA2ox7 expression was important for the regulation of endogenous GA contents. Moreover, our results suggested that depletion of $\mathrm{B}$ or $\mathrm{R}$ lights increased endogenous GA contents and may induce stem elongation. A decrease in a specific light wavelength does not occur naturally, but it can occur under artificial light conditions. Further studies, such as transcriptome analysis, are needed to better understand the mechanisms underlying the responses of the tomato to light quality and optimize the light conditions for tomato seedling production.

\section{Acknowledgments}

We thank Ms. A. Jike for technical assistance.

\section{Literature Cited}

Asahina, M., Y. Yamauchi, A. Hanada, Y. Kamiya, H. Kamada, S. Satoh and S. Yamaguchi. 2007. Effects of the removal of cotyledons on endogenous gibberellin levels in hypocotyls of young cucumber and tomato seedlings. Plant Biotechnol. 24: $99-106$

Britz, S. J. and J. C. Sager. 1990. Photomorphogenesis and photoassimilation in soybean and sorghum grown under broad spectrum or blue-deficient light sources. Plant Physiol. 94: 448-454.

Brown, C. S., A. Schuerger and J. C. Sager. 1995. Growth and photomorphogenesis of pepper plants under red LEDs with supplemental blue or far-red lighting. J. Amer. Soc. Hort. Sci. 120: 808-813.

Casal, J. J. 2012. Shade Avoidance. The Arabidopsis Book 10, e0157. DOI: 10.1199/tab.0157.

Chen, S., X. J. Wang, L. Y. Zhang, S. S. Lin, D. C. Liu, Q. Z. Wang, S. Y. Cai, R. El-Tanbouly, L. J. Gan, H. Wu and Y. Li. 
2016. Identification and characterization of tomato gibberellin 2-oxidases (GA2oxs) and effects of fruit-specific SIGA2oxl overexpression on fruit and seed growth and development. Hortic. Res. 3: 16059. DOI: 10.1038/hortres. 2016.59 .

de Wit, M., D. H. Keuskamp, F. J. Bongers, P. Hornitschek, C. M. M. Gommers, E. Reinen, C. Martínez-Cerón, C. Fankhauser and R. Pierik. 2016. Integration of phytochrome and cryptochrome signals determines plant growth during competition for light. Curr. Biol. 26: 3320-3326.

Expósito-Rodríguez, M., A. A. Borges, A. Borges-Pérez and J. A. Pérez. 2008. Selection of internal control genes for quantitative real-time RT-PCR studies during tomato development process. BMC Plant Biol. 8: 131. DOI: 10.1186/1471-22298-131.

Foo, E., J. D. Platten, J. L. Weller and J. B. Reid. 2006. PhyA and cry1 act redundantly to regulate gibberellin levels during deetiolation in blue light. Physiol. Plant. 127: 149-156.

García-Martinez, J. L. and J. Gil. 2002. Light regulation of gibberellin biosynthesis and mode of action. J. Plant Growth Regul. 20: 354-368.

Giliberto, L., G. Perrotta, P. Pallara, J. L. Weller, P. D. Fraser, P. M. Bramley, A. Fiore, M. Tavazza and G. Giuliano. 2005. Manipulation of the blue light photoreceptor cryptochrome 2 in tomato affects vegetative development, flowering time, and fruit antioxidant content. Plant Physiol. 137: 199-208.

Hedden, P. and S. G. Thomas. 2012. Gibberellin biosynthesis and its regulation. Biochem. J. 444: 11-25.

Hernández, R., T. Eguchi, M. Deveci and C. Kubota. 2016. Tomato seedling physiological responses under different percentages of blue and red photon flux ratios using LEDs and cool white fluorescent lamps. Sci. Hort. 213: 270-280.

Hirose, F., N. Inagaki, A. Hanada, S. Yamaguchi, Y. Kamiya, A. Miyao, H. Hirochika and M. Takano. 2012. Cryptochrome and phytochrome cooperatively but independently reduce active gibberellin content in rice seedlings under light irradiation. Plant Cell Physiol. 53: 1570-1582.

Honda, I., H. Matsunaga, K. Kikuchi, S. Matuo, M. Fukuda and S. Imanishi. 2017. Involvement of cytokinins, 3-indoleacetic acid, and gibberellins in early fruit growth in pepper (Capsicum annuum L.). Hortic. J. 86: 52-60.

Kotoda, N., S. Matsuo, I. Honda, K. Yano and T. Shimizu. 2016. Isolation and functional analysis of two gibberellin 20oxidase genes from satsuma mandarin (Citrus unshiu Marc.). Hortic. J. 85: 128-140.

Kozai, T. 2013. Resource use efficiency of closed plant production system with artificial light: Concept, estimation and application to plant factory. Proc. Jpn. Acad., Ser. B 89: 447461.

Kurepin, L. V. and R. P. Pharis. 2014. Light signaling and the phytohormonal regulation of shoot growth. Plant Sci. 229: 280-289.

Lin, C. T. 2002. Blue light receptors and signal transduction. Plant Cell 14: s207-s225.

Matsuo, S., K. Kikuchi, M. Fukuda, I. Honda and S. Imanishi. 2012. Roles and regulation of cytokinins in tomato fruit development. J. Exp. Bot. 63: 5569-5579.

McCree, K. J. 1972. Action spectrum, absorptance and quantum yield of photosynthesis in crop plants. Agric. Meteorol. 9: 191-216.

Mitchum, M. G., S. Yamaguchi, A. Hanada, A. Kuwahara, Y. Yoshioka, T. Kato, S. Tabata, Y. Kamiya and T. P. Sun. 2006. Distinct and overlapping roles of two gibberellin 3-oxidases in Arabidopsis development. Plant J. 45: 804-818.

Nakaune, M., A. Hanada, Y. G. Yin, C. Matsukura, S. Yamaguchi and H. Ezura. 2012. Molecular and physiological dissection of enhanced seed germination using short-term low- concentration salt seed priming in tomato. Plant Physiol. Bioch. 52: 28-37.

Nanya, K., Y. Ishigami, S. Hikosaka and E. Goto. 2012. Effects of blue and red light on stem elongation and flowering of tomato seedlings. Acta Hort. 956: 261-266.

Ninu, L., M. Ahmad, C. Miarelli, A. R. Cashmore and G. Giuliano. 1999. Cryptochrome 1 controls tomato development in response to blue light. Plant J. 18: 551-556.

Nishimura, M., T. Kozai, C. Kubota and C. Chun. 2001. Analysis of electric energy consumption and its cost for a closed-type transplant production system. J. Soc. High Technol. Agr. 13: 254-261 (In Japanese with English abstract).

Ohyama, K., T. Kozai and C. Chun. 2003. Development of closed-type transplant production system and its application. J. SHITA. 15: 1-10 (In Japanese with English abstract).

Pedmale, U. V., S. S. C. Huang, M. Zander, B. J. Cole, J. Hetzel, K. Ljung, P. A. B. Reis, P. Sridevi, K. Nito, J. R. Nery, J. R. Ecker and J. Chory. 2016. Cryptochromes interact directly with PIFs to control plant growth in limiting blue light. Cell 164: 233-245.

Rebers, M., T. Kaneta, H. Kawaide, S. Yamaguchi, Y. Y. Yang, R. Imai, H. Sekimoto and Y. Kamiya. 1999. Regulation of gibberellin biosynthesis genes during flower and early fruit development of tomato. Plant J. 17: 241-250.

Rieu, I., O. Ruiz-Rivero, N. Fernandez-Garcia, J. Griffiths, S. J. Powers, F. Gong, T. Linhartova, S. Eriksson, O. Nilsson, S. G. Thomas, A. L. Phillips and P. Hedden. 2008. The gibberellin biosynthetic genes AtGA20ox1 and AtGA20ox2 act, partially redundantly, to promote growth and development throughout the Arabidopsis life cycle. Plant J. 53: 488-504.

Serrani, J. C., O. Ruiz-Rivero, M. Fos and J. L. García-Martinez. 2008. Auxin-induced fruit-set in tomato is mediated in part by gibberellins. Plant J. 56: 922-934.

Singh, H., P. Kumar, S. Chaudhari and M. Edelstein. 2017. Tomato grafting: a global perspective. HortScience 52: 1328-1336.

van Tuinen, A., L. H. J. Kerckhoffs, A. Nagatani, R. E. Kendrick and M. Koornneef. 1995. A temporarily red light-insensitive mutant of tomato lacks a light-stable, B-like phytochrome. Plant Physiol. 108: 939-947.

Weller, J. L., G. Perrotta, M. E. L. Schreuder, A. van Tuinen, M. Koornneef, G. Giuliano and R. E. Kendrick. 2001. Genetic dissection of blue-light sensing in tomato using mutants deficient in cryptochrome 1 and phytochromes A, B1 and B2. Plant J. 25: 427-440.

Weller, J. L., M. E. L. Schreuder, H. Smith, M. Koornneef and R. E. Kendrick. 2000. Physiological interactions of phytochromes A, B1 and B2 in the control of development in tomato. Plant J. 24: 345-356.

Wollaeger, H. M. and E. S. Runkle. 2014. Growth of impatiens, petunia, salvia, and tomato seedlings under blue, green, and red light-emitting diodes. HortScience 49: 734-740.

Wollaeger, H. M. and E. S. Runkle. 2015. Growth and acclimation of impatiens, salvia, petunia, and tomato seedlings to blue and red light. HortScience 50: 522-529.

Yamaguchi, S. 2008. Gibberellin metabolism and its regulation. Annu. Rev. Plant Biol. 59: 225-251.

Yorio, N. C., G. Goins, H. Kagie, R. Wheeler and J. C. Sager. 2001. Improving spinach, radish, and lettuce growth under red LEDs with blue light supplementation. HortScience 36: 380-383.

Zhao, X. Y., X. H. Yu, E. Foo, G. M. Symons, J. Lopez, K. T. Bendehakkalu, J. Xiang, J. L. Weller, X. M. Liu, J. B. Reid and C. T. Lin. 2007. A study of gibberellin homeostasis and cryptochrome-mediated blue light inhibition of hypocotyl elongation. Plant Physiol. 145: 106-118. 